Article

\title{
Families of Integrals of Polylogarithmic Functions ${ }^{\dagger}$
}

\section{Anthony Sofo}

Victoria University, P. O. Box 14428, Melbourne City, Victoria 8001, Australia; anthony.sofo@vu.edu.au

† Lovingly dedicated to Mia Eva Manca.

Received: 30 December 2018; Accepted: 26 January 2019; Published: 3 February 2019

Abstract: We give an overview of the representation and many connections between integrals of products of polylogarithmic functions and Euler sums. We shall consider polylogarithmic functions with linear, quadratic, and trigonometric arguments, thereby producing new results and further reinforcing the well-known connection between Euler sums and polylogarithmic functions. Many examples of integrals of products of polylogarithmic functions in terms of Riemann zeta values and Dirichlet values will be given. Suggestions for further research are also suggested, including a study of polylogarithmic functions with inverse trigonometric functions.

Keywords: Euler sums; zeta functions; Dirichlet functions; series representation; harmonic number

MSC: 11M06; 11M32; 33B15

\section{Introduction and Preliminaries}

It is well known that integrals of products of polylogarithmic functions can be associated with Euler sums, see Reference [1]. In this paper, we investigate the representations of integrals of the type

$$
\begin{aligned}
& \int_{0}^{1} x^{m} \operatorname{Li}_{q}( \pm x) \mathrm{Li}_{t}( \pm x) d x, \\
& \int_{0}^{1} \frac{1}{x} \operatorname{Li}_{q}( \pm x) \mathrm{Li}_{t}\left(x^{2}\right) d x,
\end{aligned}
$$

for $m \geq-2$, and for integers $q$ and $t$. For $m=-2,-1,0$, we give explicit representations of the integrals in terms of Euler sums. For the case $\int_{0}^{1} x^{m} \operatorname{Li}_{t}(-x) \operatorname{Li}_{q}(-x) d x$, for $m \geq 0$, we show that the integral satisfies a certain recurrence relation. We also mention two specific integrals with a trigonometric argument in the polylogarithm of the form

$$
\int_{0}^{1} \sin \pi x \operatorname{Li}_{p}(\sin \pi x) d x .
$$

Some examples are highlighted, almost none of which are amenable to a computer mathematical package. This work extends the results given in Reference [1], where the author examined integrals with positive arguments of the polylogarithm. Devoto and Duke [2] also list many identities of lower order polylogarithmic integrals and their relations to Euler sums. Some other important sources of information on polylogarithm functions are the works of References [3] and [4]. In References [5] and [6], the authors explore the algorithmic and analytic properties of generalized harmonic Euler sums systematically, in order to compute the massive Feynman integrals which arise in quantum field 
theories and in certain combinatorial problems. Identities involving harmonic sums can arise from their quasi-shuffle algebra or from other properties, such as relations to the Mellin transform

$$
M[f(z)](N)=\int_{0}^{1} d z z^{N} f(z),
$$

where the basic functions $f(z)$ typically involve polylogarithms and harmonic sums of lower weight. Applying the latter type of relations, the author in Reference [7] expresses all harmonic sums of the above type with weight $w=6$, in terms of Mellin transforms and combinations of functions and constants of lower weight. In another interesting and related paper [8], the authors prove several identities containing infinite sums of values of the Roger's dilogarithm function. defined on $x \in[0,1]$, by

$$
L_{R}(x)=\left\{\begin{array}{cc}
\mathrm{Li}_{2}(x)+\frac{1}{2} \ln x \ln (1-x) ; 0<x<1 \\
0 ; & x=0 \\
\zeta(2) ; & x=1
\end{array} .\right.
$$

The Lerch transcendent,

$$
\Phi(z, t, a)=\sum_{m=0}^{\infty} \frac{z^{m}}{(m+a)^{t}}
$$

is defined for $|z|<1$ and $\Re(a)>0$ and satisfies the recurrence

$$
\Phi(z, t, a)=z \Phi(z, t, a+1)+a^{-t} .
$$

The Lerch transcendent generalizes the Hurwitz zeta function at $z=1$,

$$
\Phi(1, t, a)=\sum_{m=0}^{\infty} \frac{1}{(m+a)^{t}}
$$

and the polylogarithm, or de-Jonquière's function, when $a=1$,

$$
\operatorname{Li}_{t}(z):=\sum_{m=1}^{\infty} \frac{z^{m}}{m^{t}}, t \in \mathbb{C} \text { when }|z|<1 ; \Re(t)>1 \text { when }|z|=1 .
$$

Let

$$
H_{n}=\sum_{r=1}^{n} \frac{1}{r}=\int_{0}^{1} \frac{1-t^{n}}{1-t} d t=\gamma+\psi(n+1)=\sum_{j=1}^{\infty} \frac{n}{j(j+n)}, \quad H_{0}:=0
$$

be the $n t h$ harmonic number, where $\gamma$ denotes the Euler-Mascheroni constant,

$$
H_{n}^{(m)}=\sum_{r=1}^{n} \frac{1}{r^{m}}=\frac{(-1)^{m-1}}{(m-1) !} \int_{0}^{1}\left(\frac{1-t^{n}}{1-t}\right) \ln ^{m-1}(t) d t
$$

is the $m$ th order harmonic number and $\psi(z)$ is the digamma (or psi) function defined by

$$
\psi(z):=\frac{d}{d z}\{\log \Gamma(z)\}=\frac{\Gamma^{\prime}(z)}{\Gamma(z)} \text { and } \psi(1+z)=\psi(z)+\frac{1}{z^{\prime}}
$$

moreover,

$$
\psi(z)=-\gamma+\sum_{n=0}^{\infty}\left(\frac{1}{n+1}-\frac{1}{n+z}\right) .
$$


More generally, a non-linear Euler sum may be expressed as

$$
\sum_{n \geq 1} \frac{( \pm 1)^{n}}{n^{p}}\left(\prod_{j=1}^{t}\left(H_{n}^{\left(\alpha_{j}\right)}\right)^{q_{j}} \prod_{k=1}^{r}\left(J_{n}^{\left(\beta_{k}\right)}\right)^{m_{k}}\right)
$$

where $p \geq 2, t, r, q_{j}, \alpha_{j}, m_{k}, \beta_{k}$ are positive integers and

$$
\left(H_{n}^{(\alpha)}\right)^{q}=\left(\sum_{j=1}^{n} \frac{1}{j^{\alpha}}\right)^{q},\left(J_{n}^{(\beta)}\right)^{m}=\left(\sum_{j=1}^{n} \frac{(-1)^{j+1}}{j^{\beta}}\right)^{m} .
$$

If, for a positive integer,

$$
\lambda=\sum_{j=1}^{t} \alpha_{j} q_{j}+\sum_{j=1}^{r} \beta_{j} m_{j}+p,
$$

then we call it a $\lambda$-order Euler sum. The polygamma function

$$
\psi^{(k)}(z)=\frac{d^{k}}{d z^{k}}\{\psi(z)\}=(-1)^{k+1} k ! \sum_{r=0}^{\infty} \frac{1}{(r+z)^{k+1}}
$$

and satisfies the recurrence relation

$$
\psi^{(k)}(z+1)=\psi^{(k)}(z)+\frac{(-1)^{k} k !}{z^{k+1}} .
$$

The connection of the polygamma function with harmonic numbers is

$$
H_{z}^{(\alpha+1)}=\zeta(\alpha+1)+\frac{(-1)^{\alpha}}{\alpha !} \psi^{(\alpha)}(z+1), z \neq\{-1,-2,-3, \ldots\} .
$$

and the multiplication formula is

$$
\psi^{(k)}(p z)=\delta_{m, 0} \ln p+\frac{1}{p^{k+1}} \sum_{j=0}^{p-1} \psi^{(k)}\left(z+\frac{j}{p}\right)
$$

for $p$ is a positive integer and $\delta_{p, k}$ is the Kronecker delta. We define the alternating zeta function (or Dirichlet eta function) $\eta(z)$ as

$$
\eta(z):=\sum_{n=1}^{\infty} \frac{(-1)^{n+1}}{n^{z}}=\left(1-2^{1-z}\right) \zeta(z)
$$

where $\eta(1)=\ln 2$. If we put

$$
S(p, q):=\sum_{n=1}^{\infty} \frac{(-1)^{n+1} H_{n}^{(p)}}{n^{q}},
$$

in the case where $p$ and $q$ are both positive integers and $p+q$ is an odd integer, Flajolet and Salvy [9] gave the identity

$$
\begin{aligned}
2 S(p, q)= & \left(1-(-1)^{p}\right) \zeta(p) \eta(q)+2(-1)^{p} \sum_{i+2 k=q}\left(\begin{array}{c}
p+i-1 \\
p-1
\end{array}\right) \zeta(p+i) \eta(2 k) \\
& +\eta(p+q)-2 \sum_{j+2 k=p}\left(\begin{array}{c}
q+j-1 \\
q-1
\end{array}\right)(-1)^{j} \eta(q+j) \eta(2 k),
\end{aligned}
$$


where $\eta(0)=\frac{1}{2}, \eta(1)=\ln 2, \zeta(1)=0$, and $\zeta(0)=-\frac{1}{2}$ in accordance with the analytic continuation of the Riemann zeta function. We also know, from the work of Reference [10] that for odd weight $w=(p+q)$ we have

$$
\begin{aligned}
B W(p, q)= & \sum_{n=1}^{\infty} \frac{H_{n}^{(p)}}{n^{q}}=(-1)^{p} \sum_{j=1}^{\left[\frac{p}{2}\right]}\left(\begin{array}{c}
p+q-2 j-1 \\
p-1
\end{array}\right) \zeta(p+q-2 j) \zeta(2 j) \\
+ & \frac{1}{2}\left(1+(-1)^{p+1}\right) \zeta(p) \zeta(q)+(-1)^{p} \sum_{j=1}^{\left[\frac{p}{2}\right]}\left(\begin{array}{c}
p+q-2 j-1 \\
q-1
\end{array}\right) \zeta(p+q-2 j) \zeta(2 j) \\
& +\frac{\zeta(p+q)}{2}\left(1+(-1)^{p+1}\left(\begin{array}{c}
p+q-1 \\
p
\end{array}\right)+(-1)^{p+1}\left(\begin{array}{c}
p+q-1 \\
q
\end{array}\right)\right)
\end{aligned}
$$

where $[z]$ is the integer part of $z$. It appears that some isolated cases of $B W(p, q)$, for even weight $(p+q)$, can be expressed in zeta terms, but in general, almost certainly, for even weight $(p+q)$, no general closed form expression exits for $B W(p, q)$. Two examples with even weight are

$$
\sum_{n=1}^{\infty} \frac{H_{n}^{(2)}}{n^{4}}=\zeta^{2}(3)-\frac{1}{3} \zeta(6), \sum_{n=1}^{\infty} \frac{H_{n}^{(4)}}{n^{2}}=\frac{37}{12} \zeta(6)-\zeta^{2}(3)
$$

There are also the two general forms of identities

$$
2 \sum_{n=1}^{\infty} \frac{H_{n}^{(2 p+1)}}{n^{2 p+1}}=\zeta^{2}(2 p+1)+\zeta(4 p+2)
$$

and

$$
2 \sum_{n=1}^{\infty} \frac{H_{n}^{(2 p)}}{n^{2 p}}=\left(1-\frac{(4 p) ! B_{2 p}^{2}}{2((2 p) !)^{2} B_{4 p}}\right) \zeta(4 p)
$$

for $p \in \mathbb{N}$ and where $B_{p}$ are the signed Bernoulli numbers of the first kind. At even weight $w=8$, neither $B W(2,6)$ nor $B W(6,2)$ currently have a reduced expression in terms of zeta values and their products. Using (1) we can express

$$
B W(2,6)=\sum_{n=1}^{\infty} \frac{H_{n}^{(2)}}{n^{6}}=\frac{5}{3} \zeta(8)-\int_{0}^{1} \frac{\ln x \operatorname{Li}_{6}(x)}{1-x} d x
$$

and

$$
B W(6,2)=\sum_{n=1}^{\infty} \frac{H_{n}^{(6)}}{n^{2}}=\frac{5}{3} \zeta(8)+\frac{1}{5 !} \int_{0}^{1} \frac{\ln ^{5} x \mathrm{Li}_{2}(x)}{1-x} d x .
$$

The work in this paper extends the results of Reference [1] and later Reference [11], in which they gave identities of products of polylogarithmic functions with positive argument in terms of zeta functions. Other works, including References [12-23], cite many identities of polylogarithmic integrals and Euler sums. The following lemma was obtained by Freitas, [1]:

Lemma 1. For $q$ and t positive integers

$$
\begin{aligned}
I^{(+,+)}(q, t) & =\int_{0}^{1} \frac{L i_{t}(x) L i_{q}(x)}{x} d x \\
& =\sum_{j=1}^{q-1}(-1)^{j+1} \zeta(t+j) \zeta(q-j+1)+(-1)^{q+1} E U(t+q)
\end{aligned}
$$

where EU $(m)$ is Euler's identity given in the next lemma. 
Lemma 2. The following identities hold: for $m \in \mathbb{N}$. The classical Euler identity, as given by Euler, states

$$
\begin{aligned}
E U(m)= & \sum_{n=1}^{\infty} \frac{H_{n}}{n^{m}}=\left(\frac{m}{2}+1\right) \zeta(m+1) \\
& -\frac{1}{2} \sum_{j=1}^{m-2} \zeta(m-j) \zeta(j+1) .
\end{aligned}
$$

The following identities are new. For $p$ a positive even integer,

$$
\begin{aligned}
H E(p)= & \sum_{n=1}^{\infty} \frac{H_{n}}{(2 n+1)^{p}}=\frac{p}{2}(\zeta(p+1)+\eta(p+1))-(\zeta(p)+\eta(p)) \ln 2 \\
& -\frac{1}{2} \sum_{j=1}^{\frac{p}{2}-1}(\zeta(p+1-2 j)+\eta(p+1-2 j))(\zeta(2 j)+\eta(2 j)) .
\end{aligned}
$$

For $p$ a positive odd integer,

$$
\begin{aligned}
H O(p)= & \sum_{n=1}^{\infty} \frac{H_{n}}{(2 n+1)^{p}}=\frac{p}{4}(\zeta(p+1)+\eta(p+1))-(\zeta(p)+\eta(p)) \ln 2 \\
& -\frac{1}{4}\left(\frac{1+(-1)^{\frac{p-1}{2}}}{2}\right)\left(\zeta\left(\frac{p+1}{2}\right)+\eta\left(\frac{p+1}{2}\right)\right) \\
& -\frac{1}{2} \sum_{j=1}^{b}(\zeta(p-2 j)+\eta(p-2 j))(\zeta(2 j+1)+\eta(2 j+1))
\end{aligned}
$$

where $\eta(z)$ is the Dirichlet eta function, $b=\left[\frac{p-1}{4}\right]-\left(\frac{1+(-1)^{\frac{p-1}{2}}}{2}\right)$, and $[z]$ is the greatest integer less than z. For $p$ and t positive integers we have

$$
\begin{aligned}
F(p, t)= & \sum_{n=1}^{\infty} \frac{(-1)^{n+1}}{n^{p}(n+1)^{t}} \\
= & \sum_{r=1}^{p}(-1)^{p-r}\left(\begin{array}{c}
p+t-r-1 \\
p-r
\end{array}\right) \eta(r) \\
& +\sum_{s=1}^{t}(-1)^{p+1}\left(\begin{array}{c}
p+t-s-1 \\
t-s
\end{array}\right)(1-\eta(s)), \\
G(p, t)= & \sum_{n=1}^{\infty} \frac{1}{n^{p}(n+1)^{t}}=\left(\begin{array}{c}
-1)^{p+1}\left(\begin{array}{c}
p+t-1 \\
p
\end{array}\right) \\
\end{array}\right. \\
& +\sum_{r=2}^{p}(-1)^{p-r}\left(\begin{array}{c}
p+t-r-1 \\
p-r
\end{array}\right) \zeta(r) \\
& +\sum_{s=1}^{t}(-1)^{p}\left(\begin{array}{c}
p+t-s-1 \\
t-s
\end{array}\right) \zeta(s),
\end{aligned}
$$




$$
\begin{aligned}
H A(t, q)= & \sum_{n=1}^{\infty} \frac{(-1)^{n+1} H_{n}}{n^{t}(n+1)^{q}}=(-1)^{t+1}\left(\begin{array}{c}
q+t-2 \\
t-1
\end{array}\right)\left(\frac{1}{2} \zeta(2)-\ln ^{2} 2\right) \\
& +\sum_{r=2}^{t}(-1)^{t-r}\left(\begin{array}{c}
q+t-r-1 \\
t-r
\end{array}\right) S(1, r) \\
& +\sum_{s=2}^{q}(-1)^{t}\left(\begin{array}{c}
q+t-s-1 \\
q-s
\end{array}\right)(\eta(s+1)-S(1, s)),
\end{aligned}
$$

and

$$
\begin{aligned}
H G(p, t)= & \sum_{n=1}^{\infty} \frac{H_{n}}{n^{p}(n+1)^{t}}=(-1)^{p+1}\left(\begin{array}{c}
p+t-2 \\
p-1
\end{array}\right) \zeta(2) \\
& +\sum_{r=2}^{p}(-1)^{p-r}\left(\begin{array}{c}
p+t-r-1 \\
p-r
\end{array}\right) E U(r) \\
& +\sum_{s=2}^{t}(-1)^{p}\left(\begin{array}{c}
p+t-s-1 \\
t-s
\end{array}\right)(E U(s)-\zeta(s+1)) .
\end{aligned}
$$

Proof. The identity (8) is the Euler relation and by manipulation we arrive at (9) and (10). The results (9) and (10) are closely related to those given by Nakamura and Tasaka [24]. For the proof of (11) we notice that

$$
\begin{aligned}
\frac{1}{n^{p}(n+1)^{t}}= & \sum_{r=1}^{p}(-1)^{p-r}\left(\begin{array}{c}
p+t-r-1 \\
p-r
\end{array}\right) \frac{1}{n^{r}} \\
& +\sum_{s=1}^{t}(-1)^{p}\left(\begin{array}{c}
p+t-s-1 \\
t-s
\end{array}\right) \frac{1}{(n+1)^{s}}
\end{aligned}
$$

therefore, summing over the integers $n$,

$$
\begin{aligned}
F(p, t)= & \sum_{n=1}^{\infty} \frac{(-1)^{n+1}}{n^{p}(n+1)^{t}}=\sum_{r=1}^{p}(-1)^{p-r}\left(\begin{array}{c}
p+t-r-1 \\
p-r
\end{array}\right) \eta(r) \\
& +\sum_{s=1}^{t}(-1)^{p}\left(\begin{array}{c}
p+t-s-1 \\
t-s
\end{array}\right)(1-\eta(s))
\end{aligned}
$$

and hence (11) follows. Consider

$$
\begin{aligned}
\frac{1}{n^{p}(n+1)^{t}}= & \frac{(-1)^{p+1}}{n(n+1)}\left(\begin{array}{c}
p+t-2 \\
p-1
\end{array}\right)+\sum_{r=2}^{p}(-1)^{p-r}\left(\begin{array}{c}
p+t-r-1 \\
p-r
\end{array}\right) \frac{1}{n^{r}} \\
& +\sum_{s=2}^{t}(-1)^{p}\left(\begin{array}{c}
p+t-s-1 \\
t-s
\end{array}\right) \frac{1}{(n+1)^{s}}
\end{aligned}
$$

and summing over the integers $n$ produces the result (12). The proof of (13) and (14) follows by summing $\sum_{n=1}^{\infty} \frac{H_{n}}{n^{p}(n+1)^{t}}$ in partial fraction form. Here is an example from (10):

$$
\sum_{n=1}^{\infty} \frac{H_{n}}{(2 n+1)^{9}}=\frac{9207}{2048} \zeta(10)-\frac{961}{1024} \zeta^{2}(5)-\frac{889}{512} \zeta(7) \zeta(3)-\frac{511}{256} \zeta(9) \ln 2
$$


and from (9):

$$
\sum_{n=1}^{\infty} \frac{H_{n}}{(2 n+1)^{6}}=\frac{381}{64} \zeta(7)-\frac{93}{64} \zeta(5) \zeta(2)-\frac{105}{64} \zeta(4) \zeta(3)-\frac{63}{32} \zeta(6) \ln 2
$$

\section{Summation Identity}

We now prove the following theorems:

Theorem 1. For positive integers $q$ and $t$, the integral of the product of two polylogarithmic functions with negative arguments

$$
\begin{aligned}
I_{0}^{(-,-)}(q, t)= & \int_{0}^{1} L i_{t}(-x) L i_{q}(-x) d x=\int_{-1}^{0} L i_{t}(x) L i_{q}(x) d x \\
= & \sum_{j=1}^{q-1}(-1)^{j+1} \eta(q-j+1) F(t, j) \\
& +(-1)^{q}(F(t, q+1)-(F(t, q)-G(t, q)) \ln 2)+(-1)^{q} W_{n}(q, t)
\end{aligned}
$$

where the sum

$$
W_{n}(q, t)=\sum_{n=1}^{\infty} H_{n}\left(\frac{1}{(2 n)^{t}(2 n+1)^{q}}-\frac{1}{n^{t}(n+1)^{q}}+\frac{1}{(2 n+1)^{t}(2 n+2)^{q}}\right)
$$

is obtained from (8), (9), (10), and the terms $F(\cdot, \cdot), G(\cdot, \cdot)$ are obtained from (11) and (12), respectively.

Proof. By the definition of the polylogarithmic function, we have

$$
\begin{aligned}
I_{0}^{(-,-)}(q, t)=\int_{0}^{1} \operatorname{Li}_{t}(-x) \operatorname{Li}_{q}(-x) d x=\sum_{n=1}^{\infty} \sum_{r=1}^{\infty} \frac{(-1)^{n+r}}{n^{t} r^{q}(n+r+1)} \\
=\sum_{n=1}^{\infty} \sum_{r=1}^{\infty} \frac{(-1)^{n+r}}{n^{t}}\left(\frac{(-1)^{q}}{(n+r+1)(n+1)^{q}}+\sum_{j=1}^{q} \frac{(-1)^{j+1}}{(n+1)^{j} r^{q-j+1}}\right) \\
=\sum_{n=1}^{\infty} \frac{(-1)^{n+r}}{n^{t}}\left(\frac{(-1)^{q+1}}{(n+1)^{q}}\left(\frac{1}{2} H_{\frac{n+1}{2}}-\frac{1}{2} H_{\frac{n}{2}}\right)+\sum_{j=1}^{q} \frac{(-1)^{j+1} \eta(q-j+1)}{(n+1)^{j}}\right) \\
=\sum_{j=1}^{q-1}(-1)^{j+1} \eta(q-j+1) \sum_{n=1}^{\infty} \frac{(-1)^{n+1}}{n^{t}(n+1)^{j}}+(-1)^{q} \sum_{n=1}^{\infty} \frac{(-1)^{n+1}}{n^{t}(n+1)^{q+1}} \\
\quad+(-1)^{q} \sum_{n=1}^{\infty} \frac{(-1)^{n+1}}{n^{t}(n+1)^{q}}\left(\frac{1}{2} H_{\frac{n+1}{2}}-\frac{1}{2} H_{\frac{n}{2}}-\ln 2\right) .
\end{aligned}
$$

Now we utilize the double argument identity (3) together with (11), we obtain

$$
\begin{aligned}
I_{0}^{(-,-)}(q, t)= & \sum_{j=1}^{q-1}(-1)^{j+1} \eta(q-j+1) F(t, j)+(-1)^{q} F(t, q+1) \\
& +(-1)^{q} \sum_{n=1}^{\infty} \frac{(-1)^{n+1}}{n^{t}(n+1)^{q}}\left(H_{n}-H_{\frac{n}{2}}-2 \ln 2\right)
\end{aligned}
$$


and we can use the alternating harmonic number sum identity (5) to simplify the last sum. However, we shall simplify further as follows:

$$
\begin{aligned}
I_{0}^{(-,-)}(q, t)= & \sum_{j=1}^{q-1}(-1)^{j+1} \eta(q-j+1) F(t, j)+(-1)^{q} F(t, q+1) \\
& +(-1)^{q} \sum_{n=1}^{\infty} \frac{(-1)^{n+1}}{n^{t}(n+1)^{q}}\left((-1)^{n+1}\left(H_{\left[\frac{n}{2}\right]}-H_{n}\right)-\left(1+(-1)^{n}\right) \ln 2\right)
\end{aligned}
$$

where $[z]$ is the integer part of $z$. Now,

$$
\begin{aligned}
I_{0}^{(-,-)}(q, t)= & \sum_{j=1}^{q-1}(-1)^{j+1} \eta(q-j+1) F(t, j)+(-1)^{q} F(t, q+1) \\
& -(-1)^{q}(F(t, q)-G(t, q)) \ln 2+(-1)^{q} W_{n}(q, t)
\end{aligned}
$$

where

$$
W_{n}(q, t)=\sum_{n=1}^{\infty} H_{n}\left(\frac{1}{(2 n)^{t}(2 n+1)^{q}}-\frac{1}{n^{t}(n+1)^{q}}+\frac{1}{(2 n+1)^{t}(2 n+2)^{q}}\right)
$$

and the infinite positive harmonic number sums are easily obtainable from (8), (9), (10), hence the identity (15) is achieved.

The next theorem investigates the integral of the product of polylogarithmic functions divided by a linear function.

Theorem 2. Let $(t, q)$ be positive integers, then for $t+q$ an odd integer

$$
\begin{aligned}
I^{(-,-)}(q, t) & =\int_{0}^{1} \frac{L i_{t}(-x) L i_{q}(-x)}{x} d x=-\int_{-1}^{0} \frac{L i_{t}(x) L i_{q}(x)}{x} d x \\
& =\sum_{j=1}^{q-1}(-1)^{j+1} \eta(t+j) \eta(q-j+1)+(-1)^{q+1}(\zeta(t+q)+\eta(t+q)) \ln 2 \\
& +(-1)^{q+1}\left(2^{-t-q}-1\right) E U(q+t)+(-1)^{q+1} H O(q+t) .
\end{aligned}
$$

For $t+q$ an even integer

$$
\begin{aligned}
I^{(-,-)}(q, t)= & \sum_{j=1}^{q-1}(-1)^{j+1} \eta(t+j) \eta(q-j+1) \\
& +(-1)^{q+1}(\zeta(t+q)+\eta(t+q)) \ln 2+(-1)^{q+1}\left(2^{-t-q}-1\right) E U(q+t) \\
& +(-1)^{q+1} \operatorname{HE}(q+t) .
\end{aligned}
$$

Proof. Consider

$$
\begin{aligned}
I^{(-,-)}(q, t) & =\int_{0}^{1} \frac{\mathrm{Li}_{t}(-x) \mathrm{Li}_{q}(-x)}{x} d x \\
& =\sum_{n \geq 1} \frac{(-1)^{n}}{n^{t}} \int_{0}^{1} x^{n-1} \operatorname{Li}_{q}(-x) d x,
\end{aligned}
$$


and successively integrating by parts leads to

$$
I^{(-,-)}(q, t)=\sum_{n \geq 1} \frac{(-1)^{n}}{n^{t+j}} \sum_{j=1}^{q-1} \eta(q-j+1)+\sum_{n \geq 1} \frac{(-1)^{n+q+1}}{n^{t+q-1}} \int_{0}^{1} x^{n-1} \operatorname{Li}_{1}(-x) d x
$$

Evaluating the inner integral,

$$
\begin{gathered}
\int_{0}^{1} x^{n-1} \mathrm{Li}_{1}(-x) d x=-\int_{0}^{1} x^{n-1} \ln (1+x) d x \\
=\frac{1}{n}\left(\frac{1}{2} H_{\frac{n}{2}}-\frac{1}{2} H_{\frac{n-1}{2}}-\ln 2\right),
\end{gathered}
$$

so that

$$
\begin{aligned}
I^{(-,-)}(q, t)= & \sum_{n \geq 1} \frac{(-1)^{n}}{n^{t+j}} \sum_{j=1}^{q-1}(-1)^{j} \eta(q-j+1) \\
& +\sum_{n \geq 1} \frac{(-1)^{n+q+1}}{n^{t+q}}\left(\frac{1}{2} H_{\frac{n}{2}}-\frac{1}{2} H_{\frac{n-1}{2}}-\ln 2\right) \\
= & \sum_{j=1}^{q-1}(-1)^{j+1} \eta(q-j+1) \eta(t+j) \\
& +\sum_{n \geq 1} \frac{(-1)^{n+q+1}}{n^{t+q}}\left(\frac{1}{2} H_{\frac{n}{2}}-\frac{1}{2} H_{\frac{n-1}{2}}-\ln 2\right) .
\end{aligned}
$$

If we now utilize the multiplication formula (3), we can write

$$
I^{(-,-)}(q, t)=\sum_{j=1}^{q-1}(-1)^{j+1} \eta(q-j+1) \eta(t+j)+(-1)^{q+1} \sum_{n \geq 1} \frac{(-1)^{n+1}}{n^{t+q}}\left(H_{n}-H_{\frac{n}{2}}\right) .
$$

Now consider the harmonic number sum

$$
\begin{aligned}
& \sum_{n \geq 1} \frac{(-1)^{n+1}}{n^{t+q}}\left(H_{n}-H_{\frac{n}{2}}\right)=\sum_{n \geq 1} \frac{(-1)^{n+1}}{n^{t+q}}\left(\begin{array}{c}
\left(1-(-1)^{n}\right) \ln 2 \\
+(-1)^{n+1}\left(H_{\left[\frac{n}{2}\right]}-H_{n}\right)
\end{array}\right) \\
& =\sum_{n \geq 1} \frac{(-1)^{n+1}}{n^{t+q}}\left(\left(1-(-1)^{n}\right) \ln 2+(-1)^{n+1} \sum_{j=1}^{n} \frac{(-1)^{j}}{j}\right) \\
& =\sum_{n \geq 1} \frac{(-1)^{n+1}}{n^{t+q}}\left(1-(-1)^{n}\right) \ln 2+\sum_{n \geq 1}\left(\frac{1}{2^{t+q}}-1\right) \frac{H_{n}}{n^{t+q}}+\sum_{n \geq 1} \frac{H_{n}}{(2 n+1)^{t+q}} \\
& =(\zeta(t+q)+\eta(t+q)) \ln 2+\sum_{n \geq 1}\left(\frac{1}{2^{t+q}}-1\right) \frac{H_{n}}{n^{t+q}}+\sum_{n \geq 1} \frac{H_{n}}{(2 n+1)^{t+q}}
\end{aligned}
$$


where $[z]$ is the integer part of $z$. Hence,

$$
\begin{aligned}
I^{(-,-)}(q, t)= & \sum_{j=1}^{q-1}(-1)^{j+1} \eta(q-j+1) \eta(t+j)+(-1)^{q+1}(\zeta(t+q)+\eta(t+q)) \ln 2 \\
& +(-1)^{q+1} \sum_{n \geq 1}\left(\frac{1}{2^{t+q}}-1\right) \frac{H_{n}}{n^{t+q}}+(-1)^{q+1} \sum_{n \geq 1} \frac{H_{n}}{(2 n+1)^{t+q}} \\
= & \sum_{j=1}^{q-1}(-1)^{j+1} \eta(q-j+1) \eta(t+j)+(-1)^{q+1}(\zeta(t+q)+\eta(t+q)) \ln 2 \\
& +(-1)^{q+1}\left(\frac{1}{2^{t+q}}-1\right) E U(q+t) \\
& +(-1)^{q+1}\left\{\begin{array}{l}
H O(q+t), \text { for } t+q \text { odd } \\
H E(q+t), \text { for } t+q \text { even }
\end{array}\right.
\end{aligned}
$$

hence (17) and (18) follow.

Remark 1. It is interesting to note that for $m \in \mathbb{R}$,

$$
\int_{0}^{1} \frac{L i_{t}\left(-x^{m}\right) L i_{q}\left(-x^{m}\right)}{x} d x=\frac{1}{m} \int_{0}^{1} \frac{L i_{t}(-x) L i_{q}(-x)}{x} d x .
$$

The next theorem investigates the integral of the product of polylogarithmic functions divided by a quadratic factor.

Theorem 3. For positive integers $q$ and $t$, the integral of the product of two polylogarithmic functions with negative arguments

$$
\begin{aligned}
I_{2}^{(-,-)}(q, t)= & \int_{0}^{1} \frac{L i_{t}(-x) L i_{q}(-x)}{x^{2}} d x=\int_{-1}^{0} \frac{L i_{t}(x) L i_{q}(x)}{x^{2}} d x \\
= & \eta(q+1)+\sum_{j=1}^{q-1}(-1)^{j} \eta(q-j+1) F(j, t) \\
& +(-1)^{q}(F(q, t)+G(q, t)) \ln 2+(-1)^{q} W_{n}(t, q)
\end{aligned}
$$

where the sum

$$
W_{n}(t, q)=\sum_{n=1}^{\infty} H_{n}\left(\frac{1}{(2 n)^{q}(2 n+1)^{t}}-\frac{1}{n^{q}(n+1)^{t}}+\frac{1}{(2 n+1)^{q}(2 n+2)^{t}}\right)
$$

is obtained from (8), (9), (10), and the terms $F(\cdot, \cdot), G(\cdot, \cdot)$ are obtained from (11) and (12), respectively.

Proof. Follows the same process as in Theorem 2.

The following recurrence relation holds for the reduction of the integral of the product of polylogarithmic functions multiplied by the power of its argument. 
Lemma 3. For $(q, t) \in \mathbb{N}$ and $m \geq 0$, let

$$
J(m, q, t)=\int_{0}^{1} x^{m} L i_{t}(-x) L i_{q}(-x) d x=(-1)^{m} \int_{-1}^{0} x^{m} L i_{t}(x) L i_{q}(x) d x
$$

then

$$
(m+1) J(m, q, t)=\eta(q) \eta(t)-J(m, q, t-1)-J(m, q-1, t) .
$$

For $q=1$,

$$
\begin{aligned}
(m+1) J(m, 1, t)= & \eta(t)+m J(m-1,1, t)+J(m-1,1, t-1)-J(m, 1, t-1) \\
& -m K(m, t)-K(m, t-1)
\end{aligned}
$$

where

$$
K(m, t)=\int_{0}^{1} x^{m} L i_{t}(-x) d x
$$

Proof. The proof of the lemma follows in a straightforward manner after integration by parts.

Theorem 4. For positive integers $q$ and $t$, the integral

$$
\begin{aligned}
I_{0}^{(+,-)}(q, t)= & \int_{0}^{1} L i_{q}(x) L i_{t}(-x) d x=\int_{-1}^{0} \operatorname{Li} i_{q}(-x) L i_{t}(x) d x \\
= & \sum_{j=1}^{q-1}(-1)^{j} \zeta(q-j+1) F(t, j) \\
& +(-1)^{q} F(t, q+1)+(-1)^{q} \operatorname{HA}(t, q) .
\end{aligned}
$$

Let $(q, t)$ be positive integers, then for $t+q$ an even integer,

$$
\begin{aligned}
I^{(+,-)}(q, t) & =\int_{0}^{1} \frac{L i_{q}(x) L i_{t}(-x)}{x} d x=-\int_{-1}^{0} \frac{L i_{q}(-x) L i_{t}(x)}{x} d x \\
& =\sum_{j=1}^{q-1}(-1)^{j} \eta(t+j) \zeta(q-j+1)+(-1)^{q} S(1, q+t), \\
I^{(-,+)}(q, t) & =\int_{0}^{1} \frac{L i_{q}(-x) L i_{t}(x)}{x} d x=-\int_{-1}^{0} \frac{\operatorname{Li} i_{q}(x) L i_{t}(-x)}{x} d x \\
& =\sum_{j=1}^{t-1}(-1)^{j} \eta(q+j) \zeta(t-j+1)+(-1)^{t} S(1, q+t)
\end{aligned}
$$


For positive integers $q$ and $t$,

$$
\begin{aligned}
I_{2}^{(+,-)}(q, t)= & \int_{0}^{1} \frac{L i_{q}(x) L i_{t}(-x)}{x^{2}} d x=\int_{-1}^{0} \frac{L i_{q}(-x) L i_{t}(x)}{x^{2}} d x \\
= & -\zeta(q+1)+\sum_{j=1}^{q-1}(-1)^{j+1} \zeta(q-j+1) F(j, t) \\
& +(-1)^{q+1} H A(q, t),
\end{aligned}
$$

where the terms $F(\cdot, \cdot), H A(\cdot, \cdot)$ and $S(\cdot, \cdot)$ are obtained from (11) (13) and (5), respectively.

Proof. The proof is similar to the one outlined in the previous theorems.

Theorem 5. For positive integers $q$ and $t$, the integral of the product of two polylogarithmic functions,

$$
\begin{aligned}
U^{(+,+)}(q, t)= & \int_{0}^{1} \frac{L i_{q}(x) L i_{t}\left(x^{2}\right)}{x} d x \\
= & 2^{t-1}\left(\sum_{j=1}^{q-1}(-1)^{j+1} \zeta(t+j) \zeta(q-j+1)+(-1)^{q+1} E U(t+q)\right) \\
& +2^{t-1}\left(\sum_{j=1}^{q-1}(-1)^{j} \eta(t+j) \zeta(q-j+1)+(-1)^{q} S(1, q+t)\right)
\end{aligned}
$$

where the terms $\mathrm{EU}(\cdot, \cdot), S(\cdot, \cdot)$ are obtained from (8) and (5), respectively.

Proof. By the definition of the polylogarithmic function, we have

$$
U^{(+,+)}(q, t)=\int_{0}^{1} \frac{\operatorname{Li}_{q}(x) \operatorname{Li}_{t}\left(x^{2}\right)}{x} d x=\sum_{n=1}^{\infty} \frac{1}{n^{t}} \int_{0}^{1} x^{2 n-1} \operatorname{Li}_{q}(x) d x .
$$

Successively integrating by parts leads to

$$
\begin{aligned}
U^{(+,+)}(q, t)= & \sum_{j=1}^{q-1}(-1)^{j+1} \zeta(q-j+1) \sum_{n \geq 1} \frac{1}{n^{t}(2 n)^{j}} \\
& +\sum_{n \geq 1} \frac{(-1)^{q+1}}{n^{t}(2 n)^{q-1}} \int_{0}^{1} x^{2 n-1} \operatorname{Li}_{1}(x) d x, \\
= & \sum_{j=1}^{q-1}(-1)^{j+1} \zeta(q-j+1) \sum_{n \geq 1} \frac{1}{n^{t}(2 n)^{j}}+\sum_{n \geq 1} \frac{(-1)^{q+1} H_{2 n}}{n^{t}(2 n)^{q}} \\
= & \sum_{j=1}^{q-1} \frac{(-1)^{j+1}}{2^{j}} \zeta(q-j+1) \zeta(t+j)+\frac{(-1)^{q+1}}{2^{q}} \sum_{n \geq 1} \frac{H_{2 n}}{n^{t+q}} .
\end{aligned}
$$

Alternatively, we have the relation

$$
\mathrm{Li}_{t}\left(x^{2}\right)=2^{t-1}\left(\mathrm{Li}_{t}(x)+\mathrm{Li}_{t}(-x)\right),
$$

from which we obtain

$$
\begin{aligned}
\int_{0}^{1} \frac{\mathrm{Li}_{q}(x) \mathrm{Li}_{t}\left(x^{2}\right)}{x} d x= & 2^{t-1} \int_{0}^{1} \frac{\operatorname{Li}_{q}(x)\left(\operatorname{Li}_{t}(x)+\mathrm{Li}_{t}(-x)\right)}{x} d x \\
= & 2^{t-1}\left(I^{(+,+)}(q, t)+I^{(+,-)}(q, t)\right) \\
= & 2^{t-1}\left(\sum_{j=1}^{q-1}(-1)^{j+1} \zeta(t+j) \zeta(q-j+1)+(-1)^{q+1} E U(t+q)\right) \\
& +2^{t-1}\left(\sum_{j=1}^{q-1}(-1)^{j} \eta(t+j) \zeta(q-j+1)+(-1)^{q} S(1, q+t)\right),
\end{aligned}
$$


hence the identity (23) is achieved. An alternative expansion of the integral

$$
U^{(+,+)}(q, t)=\int_{0}^{1} \frac{\operatorname{Li}_{q}(x) \operatorname{Li}_{t}\left(x^{2}\right)}{x} d x=\sum_{n \geq 1} \frac{1}{n^{q}} \sum_{r \geq 1} \frac{1}{r^{t}(n+2 r)} .
$$

By partial fraction expansion,

$$
\begin{aligned}
U^{(+,+)}(q, t)= & \sum_{n \geq 1} \frac{1}{n^{q}} \sum_{r \geq 1} \frac{1}{r^{t}(n+2 r)}=(-1)^{t-1} 2^{t-1} \sum_{n \geq 1} \frac{H_{\frac{n}{2}}}{n^{t+q}} \\
& +\sum_{j=1}^{t-1}(-1)^{j+1} 2^{j-1} \zeta(q+j) \zeta(t-j+1) .
\end{aligned}
$$

Remark 2. We note that from (23) and (24), we are able to isolate a new Euler identity of weight $q+t+1$, in the form

$$
W(a, k)=\sum_{n \geq 1} \frac{H_{a n}}{n^{k}} .
$$

For $a=2, k=q+t$

$$
\begin{aligned}
W(2, q+t)= & \sum_{n \geq 1} \frac{H_{2 n}}{n^{t+q}}=(-1)^{q+1} 2^{q+t-1}\left(I^{(+,+)}(q, t)+I^{(+,-)}(q, t)\right) \\
& -\sum_{j=1}^{q-1}(-1)^{j+q} 2^{q-j} \zeta(q-j+1) \zeta(t+j) .
\end{aligned}
$$

Similarly, from (26) and (27), we obtain, for $a=\frac{1}{2}, k=q+t$, another new Euler identity

$$
\begin{aligned}
W\left(\frac{1}{2}, q+t\right)= & \sum_{n \geq 1} \frac{H_{n}}{n^{t+q}}=(-1)^{t+1}\left(I^{(+,+)}(q, t)+I^{(+,-)}(q, t)\right) \\
& +(-1)^{t} \sum_{j=1}^{t-1}(-1)^{j+1} 2^{j-1} \zeta(q+j) \zeta(t-j+1) .
\end{aligned}
$$

The difference of (28) and (29) delivers the delightful identity

$$
\begin{aligned}
\sum_{n \geq 1} \frac{1}{n^{t+q}}\left((-1)^{t} 2^{t-1} H_{\frac{n}{2}}-\frac{(-1)^{q}}{2^{q}} H_{2 n}\right)= & \sum_{j=1}^{q-1} \frac{(-1)^{j}}{2^{j}} \zeta(q-j+1) \zeta(t+j) \\
& +\sum_{j=1}^{t-1}(-1)^{j+1} 2^{j-1} \zeta(q+j) \zeta(t-j+1) .
\end{aligned}
$$

The next theorem investigates the integral of the product of polylogarithmic functions with one positive and one negative argument.

Theorem 6. Let $(q, t)$ be positive integers, then for $t+q$ an even integer,

$$
\begin{aligned}
U^{(-,+)}(q, t)= & \int_{0}^{1} \frac{L i_{q}(-x) L i_{t}\left(x^{2}\right)}{x} d x=\int_{-1}^{0} \frac{L i_{q}(x) L i_{t}\left(x^{2}\right)}{x} d x \\
= & 2^{t-1}\left(\sum_{j=1}^{t-1}(-1)^{j} \eta(q+j) \zeta(t-j+1)+S(1, q+t)\right) \\
& +2^{t-1}\left(\sum_{j=1}^{q-1}(-1)^{j+1} \eta(t+j) \eta(q-j+1)+(-1)^{q+1}(\zeta(t+q)+\eta(t+q)) \ln 2\right) \\
& +2^{t-1}\left((-1)^{q+1}\left(2^{-t-q}-1\right) E U(t+q)+(-1)^{q+1}\left\{\begin{array}{c}
H O(q+t), \text { for } q+\text { todd } \\
H E(q+t), \text { for } q+\text { teven }
\end{array}\right) .\right.
\end{aligned}
$$


Proof. From (25), we have

$$
\begin{aligned}
U^{(-,+)}(q, t) & =\int_{0}^{1} \frac{\mathrm{Li}_{q}(-x) \mathrm{Li}_{t}\left(x^{2}\right)}{x} d x=2^{t-1} \int_{0}^{1} \frac{\operatorname{Li}_{q}(-x)\left(\mathrm{Li}_{t}(x)+\mathrm{Li}_{t}(-x)\right)}{x} d x \\
& =2^{t-1}\left(I^{(-,+)}(q, t)+I^{(-,-)}(q, t)\right)
\end{aligned}
$$

replacing $I^{(-,+)}(q, t)$, and $I^{(-,-)}(q, t)$, with (22) and (17), respectively, leads to (17), as required. We can also consider

$$
\begin{aligned}
U^{(-,+)}(q, t) & =\int_{0}^{1} \frac{\mathrm{Li}_{q}(-x) \mathrm{Li}_{t}\left(x^{2}\right)}{x} d x \\
& =\sum_{n \geq 1} \frac{(-1)^{n}}{n^{q}} \int_{0}^{1} x^{n-1} \operatorname{Li}_{t}\left(x^{2}\right) d x
\end{aligned}
$$

and successively integrating by parts, as in Theorem 1, leads to

$$
U^{(-,+)}(q, t)=\sum_{j=1}^{t-1}(-1)^{j} \zeta(t-j+1) \eta(q+j)+(-1)^{t} \sum_{n \geq 1} \frac{(-1)^{n+1} H_{\frac{n}{2}}}{n^{q+t}} .
$$

Another expansion of

$$
\begin{aligned}
U^{(-,+)}(q, t) & =\int_{0}^{1} \frac{\mathrm{Li}_{q}(-x) \mathrm{Li}_{t}\left(x^{2}\right)}{x} d x=\int_{0}^{1} \sum_{r \geq 1} \sum_{n \geq 1} \frac{(-1)^{n} x^{n+2 r-1}}{n^{q} r^{t}} d x \\
& =\sum_{r \geq 1} \sum_{n \geq 1} \frac{(-1)^{n}}{n^{q} r^{t}(n+2 r)} .
\end{aligned}
$$

Partial fraction expansion, and summing

$$
\begin{gathered}
U^{(-,+)}(q, t)=(-1)^{q} \sum_{j=1}^{q-1}(-1)^{j} 2^{j-q} \eta(1+j) \zeta(q+t-j) \\
+\frac{(-1)^{q+1}}{2^{q}} \sum_{n \geq 1}\left(\frac{H_{n}}{n^{q+t}}-\frac{H_{2 n}}{n^{q+t}}\right), \\
=(-1)^{q} \sum_{j=1}^{q-1}(-1)^{j} 2^{j-q} \eta(1+j) \zeta(q+t-j)+\frac{(-1)^{q+1}}{2^{q}}(E U(q+t)+W(2, q+t)) .
\end{gathered}
$$

hence (17) follows. We note that from (18) and (32), we obtain another new Euler sum, namely

$$
\begin{aligned}
\sum_{n \geq 1} \frac{(-1)^{n+1} H_{\frac{n}{2}}}{n^{q+t}}= & (-1)^{t}\left(I^{(-,+)}(q, t)+I^{(-,-)}(q, t)\right) \\
& +(-1)^{t} \sum_{j=1}^{t-1}(-1)^{j+1} 2^{j-t} \zeta(t-j+1) \eta(q+j) .
\end{aligned}
$$

Example 1. Some examples follow.

$$
U^{(+,+)}(3,5)=\int_{0}^{1} \frac{L i_{3}(x) L i_{5}\left(x^{2}\right)}{x} d x=\frac{521}{32} \zeta(9)-8 \zeta(2) \zeta(7)-2 \zeta(4) \zeta(5)-\frac{1}{2} \zeta(6) \zeta(3) .
$$




$$
\begin{gathered}
U^{(-,+)}(4,4)=\int_{0}^{1} \frac{L i_{4}(-x) L i_{4}\left(x^{2}\right)}{x} d x=\frac{501}{64} \zeta(9)-4 \zeta(2) \zeta(7)-\frac{11}{8} \zeta(4) \zeta(5) . \\
W(2,6+4)=\sum_{n \geq 1} \frac{H_{2 n}}{n^{10}}=\frac{2059}{4} \zeta(11)-256 \zeta(9) \zeta(2) \\
-4 \zeta(8) \zeta(3)-64 \zeta(7) \zeta(4)-16 \zeta(6) \zeta(5) . \\
W(2,2+1)=\sum_{n \geq 1} \frac{H_{2 n}}{n^{3}}=5 \zeta(4)-4 L(3)
\end{gathered}
$$

where

$$
\begin{gathered}
L(3)=\frac{11}{4} \zeta(4)-\frac{7}{4} \zeta(3) \ln 2+\frac{1}{2} \zeta(2) \ln ^{2} 2-\frac{1}{12} \ln ^{4} 2-2 L i_{4}\left(\frac{1}{2}\right) . \\
\sum_{n \geq 1} \frac{1}{n^{9}}\left(2^{3} H_{\frac{n}{2}}+\frac{1}{2^{5}} H_{2 n}\right)=\frac{17}{4} \zeta(3) \zeta(7)+\zeta^{2}(5)-\frac{517}{32} \zeta(10) . \\
W\left(\frac{1}{2}, 5+3\right)=\sum_{n \geq 1} \frac{H_{\frac{n}{2}}}{n^{8}}=\frac{521}{512} \zeta(9)-\frac{1}{64} \zeta(2) \zeta(7) \\
-\frac{1}{16} \zeta(4) \zeta(5)-\frac{1}{4} \zeta(3) \zeta(6) . \\
\sum_{n \geq 1} \frac{(-1)^{n+1} H_{\frac{n}{2}}}{n^{6}}=\frac{49}{32} \zeta(4) \zeta(3)+\frac{31}{32} \zeta(5) \zeta(2)-\frac{635}{128} \zeta(7) .
\end{gathered}
$$

The next two results deal with Polylog integrals involving the inverse of the argument.

Theorem 7. For positive integers $q$ and $t$, the integral of the product of two polylogarithmic functions,

$$
\begin{aligned}
I_{3}(q, t)= & \int_{0}^{1} \frac{L i_{q}\left(-\frac{1}{x}\right) L i_{t}\left(x^{2}\right)}{x} d x \\
= & -\frac{1}{2^{q+1}} \zeta(q+t+1)+\frac{1}{2^{q}}(E U(q+t)-W(2, q+t)) \\
& -\sum_{j=1}^{q-1} \frac{1}{2^{j}} \zeta(q+t) \eta(q-j+1)
\end{aligned}
$$

where the terms $\operatorname{EU}(\cdot), W(\cdot, \cdot)$ are obtained from (8) and (28), respectively. For positive integers $q$ and $t$, the integral of the product of two polylogarithmic functions,

$$
\begin{aligned}
I_{4}(q, t)= & \int_{0}^{1} \frac{L i_{q}\left(\frac{1}{x^{2}}\right) L i_{t}(x)}{x} d x \\
= & -2^{q} \zeta(q+t+1)+2^{q-1} W\left(\frac{1}{2}, q+t\right) \\
& +\sum_{j=1}^{q-1} 2^{j-1} \zeta(j+t) \zeta(q-j+1)-2^{q-1} i \pi \zeta(q+t)
\end{aligned}
$$

where $W\left(\frac{1}{2}, q+t\right)$ is obtained from (29), and $i=\sqrt{-1}$.

Proof. Following the same process as in Theorem 2, we have

$$
I_{3}(q, t)=\int_{0}^{1} \frac{\mathrm{Li}_{q}\left(-\frac{1}{x}\right) \mathrm{Li}_{t}\left(x^{2}\right)}{x} d x=\sum_{n \geq 1} \frac{1}{n^{t}} \int_{0}^{1} x^{2 n-1} \operatorname{Li}_{q}\left(-\frac{1}{x}\right) d x .
$$


Integrating by parts, as in Theorem 2, we have

$$
\begin{aligned}
I_{3}(q, t) & =-\sum_{j=1}^{q-1} \frac{1}{2^{j}} \eta(q-j+1) \zeta(t+j)+\sum_{n \geq 1} \frac{1}{n^{t}(2 n)^{q-1}} \int_{0}^{1} x^{2 n-1} \operatorname{Li}_{1}\left(-\frac{1}{x}\right) d x \\
& =-\sum_{j=1}^{q-1} \frac{1}{2^{j}} \eta(q-j+1) \zeta(t+j)-\frac{1}{2^{q}} \zeta(t+q)+\frac{1}{2^{q}} \sum_{n \geq 1} \frac{1}{n^{t+q}}\left(\frac{1}{2} H_{n}-\frac{1}{2} H_{n-\frac{1}{2}}-\ln 2\right),
\end{aligned}
$$

from the multiplication Formula (3), we can simplify so that

$$
I_{3}(q, t)=-\sum_{j=1}^{q-1} \frac{1}{2^{j}} \eta(q-j+1) \zeta(t+j)-\frac{1}{2^{q}} \zeta(t+q)-\frac{1}{2^{q}} \sum_{n \geq 1} \frac{1}{n^{t+q}}\left(H_{2 n}-H_{n}\right)
$$

and the proof of the first part of the theorem is finalized. The second result is proved as in Theorem 2.

\section{Example 2.}

$$
\begin{gathered}
I_{3}(4,2)=\int_{0}^{1} \frac{L i_{4}\left(-\frac{1}{x}\right) L i_{2}\left(x^{2}\right)}{x} d x=\frac{119}{64} \zeta(7)-\zeta(2) \zeta(5)-\frac{7}{16} \zeta(4) \zeta(3) . \\
I_{4}(3,5)=\int_{0}^{1} \frac{L i_{3}\left(\frac{1}{x^{2}}\right) L i_{5}(x)}{x} d x=\frac{31}{16} \zeta(2) \zeta(7)-\frac{503}{128} \zeta(9) \\
-\frac{1}{4} \zeta(4) \zeta(5)-i \pi \zeta(8) .
\end{gathered}
$$

\section{Some Extensions}

Some other valuable research on the representation of integrals of polylogarithmic functions with a trigonometric argument has been carried out, and we highlight the following results: Espinosa and Moll [25,26] give for $p \in \mathbb{N}_{0}$

$$
(p+1) \int_{0}^{1} x^{p} \ln (\sin (\pi x)) d x=-\ln 2+\sum_{j=1}^{\left[\frac{p+1}{2}\right]}(-1)^{j}\left(\begin{array}{c}
p+1 \\
2 j
\end{array}\right) \frac{(2 j) !}{(2 \pi)^{2 j}} \zeta(2 j+1)
$$

where $[m]$ is the integer part of $m$. For a non-trigonometric argument, the work of Espinosa and Moll may be extended in the following way: For $(p, q) \in \mathbb{N}$, let

$$
M(p, q)=\int_{0}^{1}\left(\frac{\ln x}{1-x}\right)^{q} \frac{\operatorname{Li}_{p}(x)}{x} d x
$$

Rewriting

$$
\frac{1}{(1-x)^{q}}=\sum_{n \geq 0}\left(\begin{array}{c}
n+q-1 \\
n
\end{array}\right) x^{n}
$$

then after integration and simplification

$$
\frac{(-1)^{q}}{q !} M(p, q)=\zeta(p+q+1)+\sum_{j \geq 1} \sum_{n \geq 1}\left(\begin{array}{c}
n+q-1 \\
n
\end{array}\right) \frac{1}{j^{p}(j+n)^{q+1}},
$$


which results in the product of zeta functions. For the case of $q=2$, we may write

$$
\begin{aligned}
& M(p, 2)=\int_{0}^{1}\left(\frac{\ln x}{1-x}\right)^{2} \frac{\operatorname{Li}_{p}(x)}{x} d x=2 \zeta(p+3)-2 \zeta(p+3) \zeta(p-1) \\
& +2 \zeta(p)(\zeta(2)+\zeta(3))-2 B W(2, p)-2 B W(3, p)+2 B W(3, p-1),
\end{aligned}
$$

where $B W(\cdot, \cdot)$ is given by $(6)$. For $p=4$, we find

$$
\begin{aligned}
M(4,2)= & \int_{0}^{1}\left(\frac{\ln x}{1-x}\right)^{2} \frac{\mathrm{Li}_{4}(x)}{x} d x=\frac{31}{16} \zeta(6)-3 \zeta^{2}(3) \\
& +20 \zeta(2) \zeta(5)+2 \zeta(3) \zeta(4)-34 \zeta(7)
\end{aligned}
$$

Choi $[27,28]$ has also produced many interesting examples of log-sine and log-cosine integrals. Mezo [29] continues the study and considers the class of integrals

$$
I_{p}=\int_{0}^{1} \operatorname{Li}_{p}(\sin \pi x) d x \text { and } J_{p}=\int_{0}^{1} \operatorname{Li}_{p}(\cos \pi x) d x
$$

such that

$$
J_{p}=\frac{1}{2^{p}} \sum_{j \geq 1}\left(\begin{array}{c}
2 j \\
j
\end{array}\right) \frac{1}{4^{j} j^{p}}
$$

and

$$
I_{p}=J_{p}+\frac{2}{\pi} \sum_{j \geq 1} \frac{(2 j-2) ! !}{(2 j-1) ! !} \frac{1}{(2 j-1)^{p}}
$$

where

$$
m ! !=\left\{\begin{array}{c}
1, \text { for } m=-1,0 \\
m(m-2) \ldots 6.4 .2, \text { for } m>0 \text { even } \\
m(m-2) \ldots 5.3 .1, \text { for } m>0 \text { odd }
\end{array}\right.
$$

Mezo [29] gives the following examples:

$$
\int_{0}^{1} \operatorname{Li}_{3}(\sec \pi x) d x=i\left(\frac{\pi}{4} \ln ^{2} 2+\frac{\pi^{3}}{48}\right)
$$

and

$$
\int_{0}^{1} \operatorname{Li}_{3}\left(\sin ^{2} \pi x\right) d x=\int_{0}^{1} \operatorname{Li}\left(\cos ^{2} \pi x\right) d x=2 \zeta(3)-2 \zeta(2) \ln 2+\frac{4}{3} \ln ^{3} 2 .
$$

It is possible to generalize (36), so that for $m \in \mathbb{N}$

$$
\int_{0}^{1} \operatorname{Li}_{p}\left(\sin ^{2 m} \pi x\right) d x=\sum_{n \geq 1} \frac{1}{2^{2 m n} n^{p}}\left(\begin{array}{c}
2 m n \\
m n
\end{array}\right),
$$

and

$$
\int_{0}^{1} \operatorname{Li}_{4}\left(\sin ^{2} \pi x\right) d x=\frac{9}{4} \zeta(4)+2 \zeta(2) \ln ^{2} 2-4 \zeta(3) \ln 2-\frac{2}{3} \ln ^{4} 2
$$


There are a number of extensions that may be very interesting to investigate further in regard to the representation of integrals of polylogarithmic functions. It would be beneficial to study the integrals

$$
\int_{0}^{1}(\sin \pi x)^{a} \operatorname{Li}_{p}^{b}(\sin \pi x) d x, \int_{0}^{1}\left(\frac{\arcsin x}{x}\right)^{a} \operatorname{Li}_{p}^{b}\left(x^{2}\right) d x
$$

where, for instance

$$
\begin{aligned}
& \pi \int_{0}^{1} \sin \pi x \operatorname{Li}_{4}\left(\tan ^{4} \frac{\pi x}{4}\right) d x= 4 \pi \int_{0}^{\frac{1}{4}} \sin 4 \pi y \operatorname{Li}_{4}\left(\tan ^{4} \pi y\right) d y \\
&=6 \zeta(4)+32 \zeta(2) \ln 2-32 \zeta(3), \\
& \int_{0}^{1} \sin \pi x \operatorname{Li}_{4}(\sin \pi x) d x= \frac{\pi}{2}-1+\frac{1}{8} \zeta(2)+\frac{\pi}{4}(2+\ln 2) \ln 2 \\
&+\frac{1}{4 \pi} \sum_{n \geq 0} \frac{2^{n} n !}{(n+1)^{3}(2 n+3) ! !}, \\
& \int_{0}^{1} \frac{\arcsin x}{x} \operatorname{Li}_{p}\left(x^{2}\right) d x=\frac{\pi}{4} \zeta(p+1)-\frac{\pi}{4} \sum_{n \geq 1} \frac{1}{2^{2 n} n^{p+1}}\left(\begin{array}{c}
2 n \\
n
\end{array}\right),
\end{aligned}
$$

and

$$
\begin{aligned}
\frac{1}{\pi} \int_{0}^{1} \frac{\arcsin x}{x} \operatorname{Li}_{4}\left(x^{2}\right) d x= & \frac{9}{8} \zeta(4) \ln 2+\frac{1}{3} \zeta(2) \ln ^{3} 2-\frac{1}{15} \ln ^{5} 2 \\
& +\frac{1}{2} \zeta(3)\left(\zeta(2)-2 \ln ^{2} 2\right)-\frac{5}{4} \zeta(5) .
\end{aligned}
$$

We may also study

$$
\int_{0}^{1} \operatorname{Li}_{p}^{a}(\sin \pi x) \operatorname{Li}_{q}^{b}(\cos \pi x) d x
$$

and the moments

$$
\begin{aligned}
& \int_{0}^{1} x^{a} \operatorname{Li}_{p}^{b}(\sin \pi x) d x \\
& \int_{0}^{1} x^{a} \operatorname{Li}_{p}^{b}(\arcsin x) d x
\end{aligned}
$$

where $(a, b, p, q) \in \mathbb{N}$.

In this paper we have highlighted various new identities of general Euler type sums, which represent the analytical solution of integrals with polylogarithm functions containing linear, quadratic, and trigonometric type arguments. In a series of papers [7,30,31], the authors explore linear combinations of associated harmonic polylogarithms and nested harmonic numbers. The multiple zeta value data mine, computed by Blumlein et al. [32], is an invaluable tool for the evaluation of harmonic numbers, in which values with weights of twelve, for alternating sums, and weights above twenty for non-alternating sums are presented. Further areas of fruitful research are those related to trigonometric, inverse trigonometric, and linear arguments for products of integrals of polylogarithmic functions. 
Funding: This research received no external funding.

Conflicts of Interest: The author declares no conflict of interest.

\section{References}

1. Freitas, P. Integrals of polylogarithmic functions, recurrence relations, and associated Euler sums. Math. Comput. 2005, 74, 1425-1440. [CrossRef]

2. Devoto, A.; Duke, D.W. Table of integrals and formulae for Feynman diagram calculations. Riv. Nuovo Cimento 1984, 7, 1-39. [CrossRef]

3. Kölbig, K.S. Nielsen's generalized polylogarithms. SIAM J. Math. Anal. 1986, 17, 1232-1258.

4. Lewin, R. Polylogarithms and Associated Functions; Elsevier Science Ltd.: North Holland, The Netherland; New York, NY, USA, 1981.

5. Ablinger, J.; Blümlein, J.; Schneider, C. Analytic and algorithmic aspects of generalized harmonic sums and polylogarithms. J. Math. Phys. 2013, 54. [CrossRef]

6. Chavez, F.; Duhr, C. Three-mass triangle integrals and single-valued polylogarithms. J. High Energy Phys. 2012. . [CrossRef]

7. Blümlein, J. Structural Relations of Harmonic Sums and Mellin Transforms at Weight w=6. Motives, Quantum Field Theory, And Pseudodifferential Operators; American Mathematical Society: Providence, RI, USA, 2010; pp. 167-187.

8. Hoorfar, A.; Qi, F. Sums of series of Rogers dilogarithm functions. Ramanujan J. 2009, 18, 231-238. [CrossRef]

9. Flajolet, P.; Salvy, B. Euler sums and contour integral representations. Exp. Math. 1998, 7, 15-35. [CrossRef]

10. Borwein, D.; Borwein, J.M.; Girgensohn, R. Explicit evaluation of Euler sums. Proc. Edinburgh Math. Soc. 1995, 38, 277-294. [CrossRef]

11. Xu, C.; Yan, Y.; Shi, Z. Euler sums and integrals of polylogarithm functions. J. Number Theory 2016, 165, 84-108. [CrossRef]

12. Ablinger, J.; Blümlein, J. Harmonic sums, polylogarithms, special numbers, and their generalizations. In Computer Algebra in Quantum Field Theory; Texts \& Monographs in Symbolic Computation; Springer: Vienna, Austria, 2013; pp. 1-32.

13. Bailey, D.H.; Borwein, J.M. Computation and structure of character polylogarithms with applications to character Mordell-Tornheim-Witten sums. Math. Comput. 2016, 85, 295-324. [CrossRef]

14. Borwein, D.; Borwein, J.M.; Bradley, D.M. Parametric Euler sum identities. J. Math. Anal. Appl. 2006, 316, 328-338. [CrossRef]

15. Borwein, J.M.; Bradley, D.M.; Broadhurst, D.J.; Lisoněk, P. Special values of multiple polylogarithms. Trans. Am. Math. Soc. 2001, 353, 907-941. [CrossRef]

16. Borwein, J.M.; Zucker, I.J.; Boersma, J. The evaluation of character Euler double sums. Ramanujan J. 2008, 15, 377-405. [CrossRef]

17. Davydychev, A.I.; Kalmykov, M.Y. Massive Feynman diagrams and inverse binomial sums. Nuclear Phys. $B$ 2004, 699, 3-64. [CrossRef]

18. Kalmykov, M.Y.; Veretin, O. Single-scale diagrams and multiple binomial sums. Phys. Lett. B 2000, 483, 315-323. [CrossRef]

19. Sofo, A. Polylogarithmic connections with Euler sums. Sarajevo J. Math. 2016, 12, 17-32. [CrossRef]

20. Sofo, A. Integrals of logarithmic and hypergeometric functions. Commun. Math. 2016, 24, 7-22. [CrossRef]

21. Sofo, A. Quadratic alternating harmonic number sums. J. Number Theory 2015, 154, 144-159. [CrossRef]

22. Sofo, A. Integrals of polylogarithmic functions with negative argument. Acta Univ. Sapientiae Math. 2018, 10, 347-367.

23. Sofo, A. Integral identities for sums. Math. Commun. 2008, 13, 303-309.

24. Nakamura, T.; Tasaka, K. Remarks on double zeta values of level 2. J. Number Theory 2013, 133, 48-54. [CrossRef]

25. Espinosa, O.; Moll, V. On Some Integrals Involving the Hurwitz Zeta Function: Part 2. Ramanujan J. 2002, 6 , 440-468.

26. Espinosa, O.; Moll, V. On Some Integrals Involving the Hurwitz Zeta Function: Part 1. Ramanujan J. 2002, 6, 159-188. [CrossRef]

27. Choi, J. Log-sine and log-cosine integrals. Honam Math. J. 2013, 35, 137-146. [CrossRef] 
28. Choi, J.; Srivastava, H.M. The Clausen function $C l_{2}(x)$ and its related integrals. Thai J. Math. 2014, 12, 251-264.

29. Mezo, I. A family of polylog-trigonometric integrals. Ramanujan J. 2018, 46, 161-171. [CrossRef]

30. Ablinger, J.; Blümlein, J.; Schneider, C. Harmonic sums and polylogarithms generated by cyclotomic polynomials. J. Math. Phys. 2011, 52, 102301. [CrossRef]

31. Blümlein, J. Algebraic relations between harmonic sums and associated quantities. Comput. Phys. Commun. 2004, 159, 19-54.

32. Blümlein, J.; Broadhurst, D.J.; Vermaseren, J.A.M. The multiple zeta value data mine. Comput. Phys. Commun. 2010, 181, 582-625.

(C) 2019 by the author. Licensee MDPI, Basel, Switzerland. This article is an open access article distributed under the terms and conditions of the Creative Commons Attribution (CC BY) license (http://creativecommons.org/licenses/by/4.0/). 